\section{Antimicrobial mouthwash}

Chlorhexidine Gluconate Medicated Mouthwash from Blackwell Supplies is the generic equivalent of Corsodyl. Chlorhexidine gluconate is a well-proven antimicrobial agent which reduces the formation of plaque and combats gingivitis and oral candida infections.

Chlorhexidine Gluconate Mouthwash is recommended for both preand post-operative use in the rapidly expanding areas of endodontics and dental implants, where leading dental specialists are increasingly exploiting its qualities to reduce infection and minimise patient discomfort. It is available in Original, Peppermint and Aniseed flavours to meet the needs of every patient. For more information please call John Jesshop of Blackwell Supplies on 02072241457 or fax 020 72241694 .

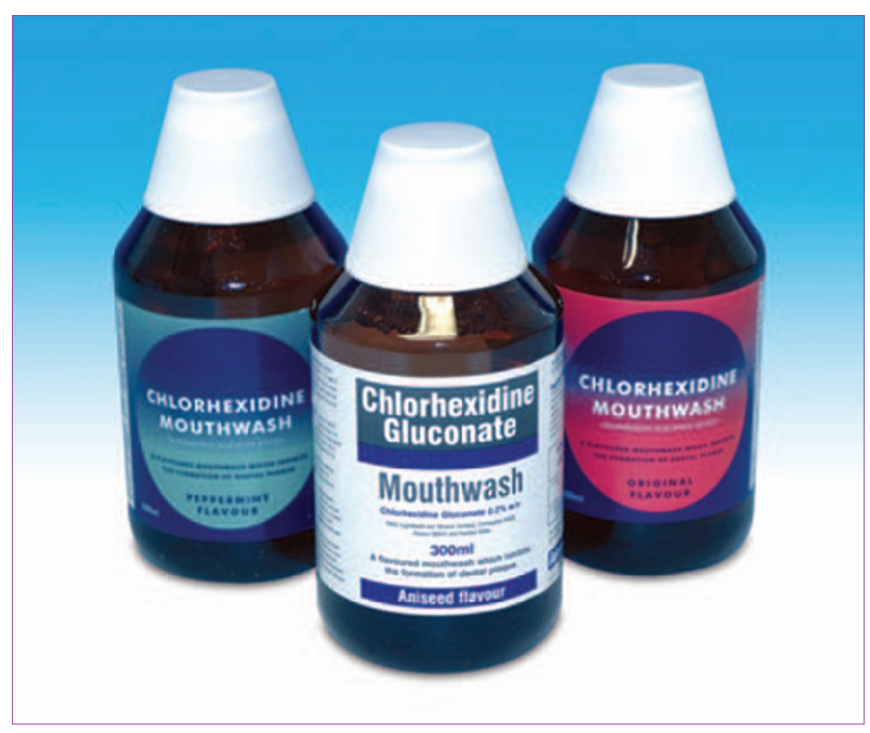

\section{Easing periodontal discomfort}

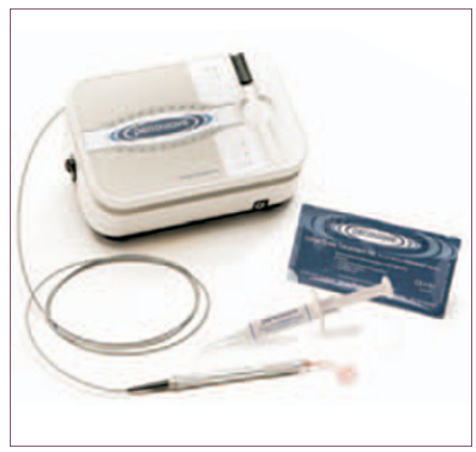

Oraldent has announced the introduction of Periowave Photodynamic Disinfection (PPD), a treatment for periodontal disease using non-thermal diode laser technology with photosensitisers to target the perio-pathogens which escape scaling and root planing (SRP).

Periowave is the ideal complement to traditional SRP and can be used during initial, supportive and maintenance therapy. Periowave is site-specific, quick and simple to administer, with each site requiring only a 60 -second treatment. Unlike antibiotic treatments, it provokes no bacterial resistance or side effects, with the additional benefit of virtually no discomfort for the patient during treatment. For further information contact 01480862080 or email enquiries@oraldent.co.uk.

\title{
Fatigue-free operation
}

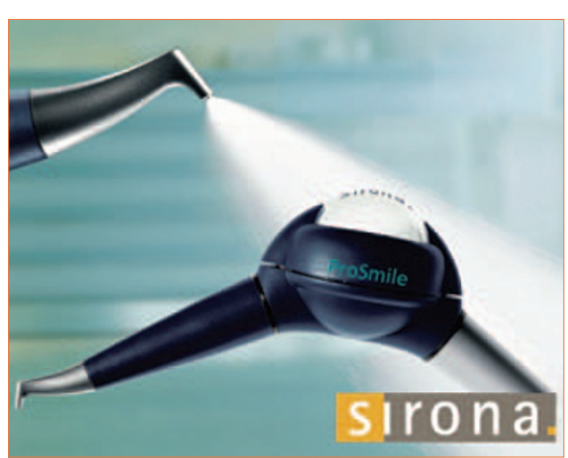

The ProSmile Handy tool from Sirona provides prophylactic air abrasive polishing for removal of plaque from all tooth surfaces. Patients can also enjoy a pleasant minty taste during treatment.

The Prosmile

Handy is completely adjustable and can be used with an interchangeable $90^{\circ}$ or $120^{\circ}$ angle spray tip for optimum access, and there is a large capacity chamber to provide adequate power supply for more than one patient.

The design of the Prosmile Handy ensures a balanced and relaxed operation by the user and can be used for the effective and minimally invasive removal of plaque from the patient.

The ProSmile Handy comes with the Sirona Click \& Go interface allowing the unit to be attached to most popular brands of handpiece couplings. It also has a stylish design to ensure a balanced feel for fatigue-free operation. For more details please contact Sirona Dental Systems Ltd 0845071504

\section{Rugged sterilising}

Stanbridge has introduced the Practice 8 and Practice 10 bench-top sterilisers that are ideal for handling pouched dental instruments. Frontloading, the Practice 8 has an eight inch stainless steel chamber capable of taking

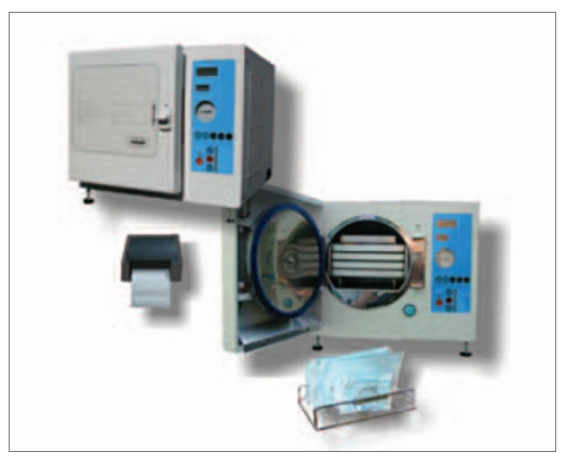
up to four trays or up to six pouches, and the pouch option conveniently sterilises instrument sets for a longer safe life.

The Practice 10 is for busy clinics. The standard unit provides downward air displacement for unwrapped instruments on trays. One option includes an integrated reservoir for auto fill. For pouching, a vacuum system pulses at the start of a cycle and pulses again at the end of a cycle to remove moisture and dry the product. This is particularly beneficial when using pouches in accordance with new healthcare regulations. A further option is a numerical record keeping system with a stand-alone printer.

The steriliser is ruggedly constructed with a stainless steel interior and a stove enamelled case, and an operating life of 20 years is expected under normal circumstances. Visit www.stanbridge.co.uk for further details. 